Document downloaded from:

http://hdl.handle.net/10251/140951

This paper must be cited as:

Rodrigo Tarrega, G.; Daros Arnau, JA.; Elena Fito, SF. (2017). Virus-host interactome: Putting the accent on how it changes. Journal of Proteomics. 156:1-4. https://doi.org/10.1016/j.jprot.2016.12.007

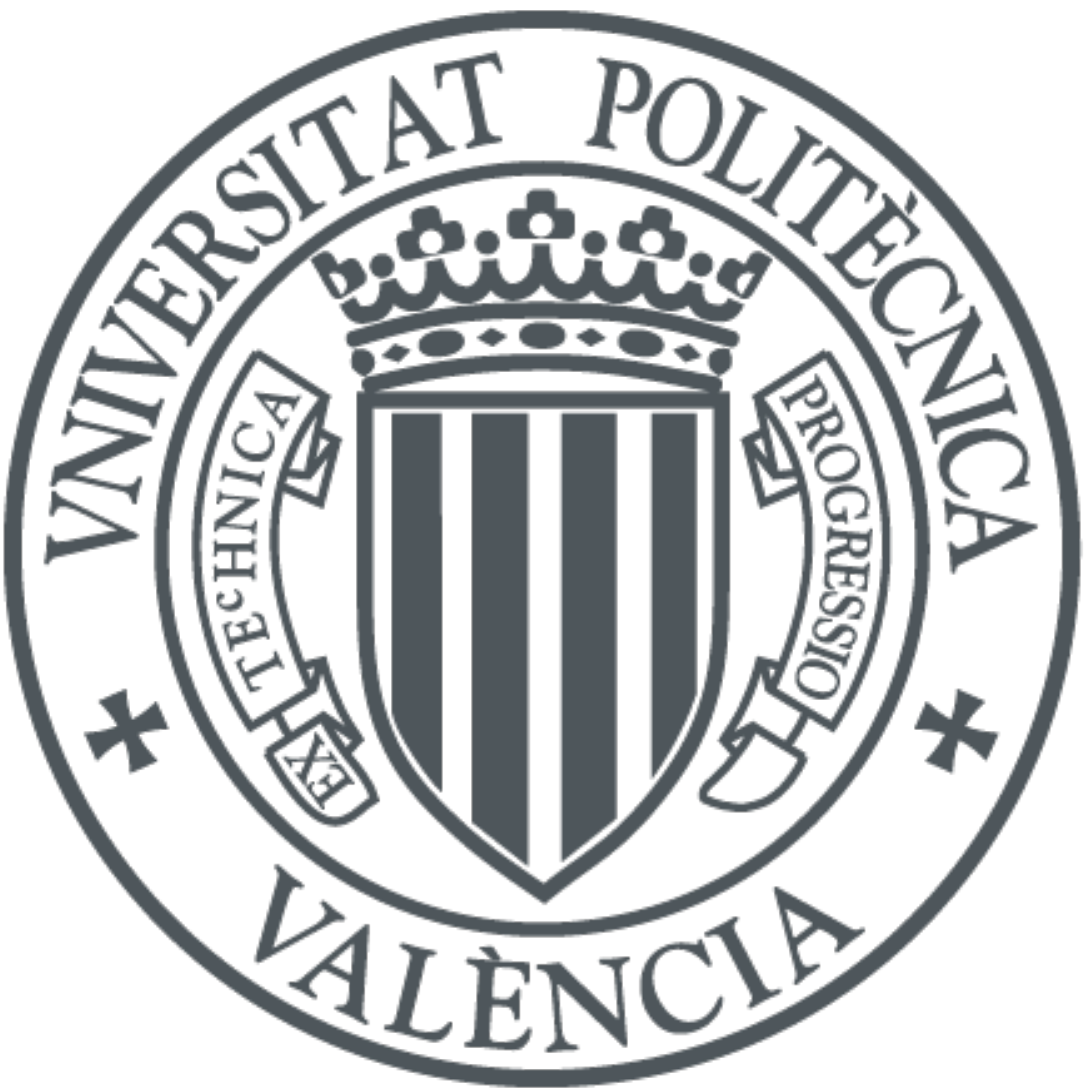

The final publication is available at

https://doi.org/10.1016/j.jprot.2016.12.007

Copyright Elsevier

Additional Information 


\title{
Virus-host interactome: Putting the accent on how it changes
}

\author{
Guillermo Rodrigo ${ }^{\mathrm{a}, \mathrm{b}, *}$, José-Antonio Daròs ${ }^{\mathrm{a}}$, Santiago F. Elena ${ }^{\mathrm{a}, \mathrm{b}, \mathrm{c}}$ \\ ${ }^{a}$ Instituto de Biología Molecular y Celular de Plantas, Consejo Superior de Investigaciones \\ Científicas - Universidad Politécnica de Valencia, 46022, Valencia, Spain \\ ${ }^{\mathrm{b}}$ Instituto de Biología Integrativa y de Sistemas, Consejo Superior de Investigaciones \\ Científicas - Universitat de València, 46980 Paterna, Spain \\ ${ }^{\mathrm{c}}$ Santa Fe Institute, Santa Fe, NM 87501, USA
}

\begin{abstract}
A b s t r a c t
Viral infections are extremely complex processes that could only be well understood by precisely characterizing the interaction networks between the virus and the host components. In recent years, much effort has gone in this direction with the aim of unveiling the molecular basis of viral pathology. These networks are mostly formed by viral and host proteins, and are expected to be dynamic both with time and space (i.e., with the progression of infection, as well as with the virus and host genotypes; what we call plastodynamic). This largely overlooked spatio-temporal evolution urgently calls for a change both in the conceptual paradigms and experimental techniques used so far to characterize virus-host interactions. More generally, molecular plasticity and temporal dynamics are unavoidable components of the mechanisms that underlie any complex disease; components whose understanding will eventually enhance our ability to modulate those networks with the aim of improving disease treatments.
\end{abstract}

Keywords: Evolution, Protein network, Systems biology, Virology

*Corresponding author at: Instituto de Biología Integrativa y de Sistemas, Consejo Superior de Investigaciones Científicas - Universitat de València, 46980 Paterna, Spain.

E-mail addresses: guillermo.rodrigo@csic.es (G. Rodrigo), jadaros@ibmcp.upv.es (J.-A. Daròs), sfelena@ibmcp.upv.es (S.F. Elena). 
Viral genomes have very limited coding capacity, and to complete their infectious cycles, viral factors (both nucleic acids and proteins) must target, directly or indirectly, many host elements. However, the interaction networks established between virus and host factors are poorly understood, and only in recent years they have been approached with the use of different experimental and computational techniques $[1,2,3]$. The construction of a molecular model that captures such virus-host interactions, as well as all interactions underlying the physiology of the host cell, is indeed the cornerstone of the emerging discipline of Systems Virology [4-7]. With this type of models in hand, it would be possible to reveal the molecular basis of disease [8], i.e., to understand how the virus reprograms the cell resources for its own benefit, and to deter-mine what counteractions viruses do to avoid host defenses $[9,10]$. Knowing where the virus-host interactions are located within these networks and what functions are being perturbed could clarify, on the one hand, general modes of viral action and, on the other hand, specific strategies of response against infection by the host cell. It is therefore expected that, in the coming years, large-scale screening techniques allow expanding considerably the list of interactors for different viruses, showing a more accurate and integrated description of viral pathogenesis at the molecular level.

However, most of the work has been (and is being) focused on characterizing a static picture of the virus-host interaction, although some pioneering attempts have addressed the dynamic properties of these interactions [11,12], also for other relevant diseases like cancer [13]. Indeed, interactions must dynamically change during the course of disease, and also among genetically heterogeneous host individuals. This is particularly serious for the case of infectious diseases, for which both the cell and the causing agent evolve during infection. Here, we introduce the term plastodynamic interactome (Fig. 1) to define the changes that a given protein interaction network experiments. These changes are an unavoidable consequence of, at least, three factors: (i) the dynamic nature of the host cell physiology and the virus life cycle, due to regulatory circuits at the molecular level, (ii) the fast evolution of viral genomes that results from a highly-efficient and error-prone polymerase, and (iii) genetic and developmental differences among individual hosts. Arguably, plasticity arises from contextual changes, either in form of expression regulation or structural modification, due to time or space, that impinge on the activity of the corresponding proteins, then remodeling the interactome [13]. Certainly, the plastodynamic nature of virus-host interactomes has not been tackled in a comprehensive way so far, despite having a deep impact in pathology and the emergence of diseases. Note that nothing prevents the ap-plication of the concept to other diseases, not necessarily of infectious etiology.

Firstly, it is clear that as long as the virus infects the host organism, it differentially expresses and rearranges its components to maximize its fitness, as it is the case, e.g., of the Human immunodeficiency virus (HIV). There, early and late transcriptional phases were identified, where small messenger RNAs were first synthesized [14]. This undoubtedly ends in a temporal program of the HIV proteome. The available proteins of the host cell to interact with the virus can also change throughout infection time, either due to differential expressions or post-translational modifications. For instance, as HIV progresses, the Vif protein induces the phosphorylation multiple host factors, leading to proteasomal degradation of immuneresponse proteins like APOBEC3C or PPP2R5A [12]. It is thus expected some variation in the virus-host interactome with time. We can distinguish between variations in short times, i.e., days or weeks post-infection, and variations in longer times, i.e., once the virus has colonized the whole organism. Secondly, mutations in the viral genome generally result in alterations in virus fitness $[15,16,17]$. RNA viruses use error-prone polymerases to produce very large progeny populations, allowing the generation of huge amounts of genetic 
variability in short periods of time, generally known as viral quasispecies [18]. This may result in a series of mutated viral proteins with differences in activity. It is presumed that the do-mains affected by these mutations, even by a single amino acid replace-ment, impaired their ability to interact with their host partners. This is the case, e.g., of the Hepatitis $C$ virus nonstructural protein 5A, where a single mutation can drastically impair the virus replication by disrupting the interaction with the human protein FKBP8 [19]. With some mutations, viral proteins may however gain new partners. Thirdly, the presence of a common virus in different hosts (even hosts of the same species but with different genotypes) will result in partially different virus-host interactomes, since the host proteins may differ in sequence and present a high degree of plasticity in their binding ability [20,21]. A differential level of expression of the very same protein among hosts could also matter in the establishment of a given interaction [22]. Moreover, environmental effects that affect the host physiology could also be included in this picture as a source of non-genetic variability among hosts. In sum, this plethora of interactions between highly polymorphic and evolvable viral populations and genetically di-verse and plastic hosts has obvious impact to understand the emergence and epidemiology of viral infections.

Even though, a precise understanding of the virus-host interplay goes beyond capturing protein interactions between virus and host factors, even beyond capturing the plastodynamic nature of such interactions. On the one hand, we would need to simultaneously retrace the mechanisms by which changes in the virus-host interactome occur. This is clearly due to a fine-tuned and highly-wired host regulatory circuitry, which can still be manipulated by the virus. Whilst defense genes are transcriptionally activated upon virus entry into the cell [23], post-translational modifications such as ubiquitination/SUMOylation [24] or phosphorylation [12] are strategies that viruses employ to remodel cell signaling with the aim of maximizing their fitness. On the other hand, we would need to take into account the subcellular localization of the virus-host protein complexes. This information is instrumental to determine with precision the mode of action of the virus, including its replication [19] and its movement through the host [25], and ultimately resolve the pathological outcome of infection.

To assess the changes in virus-host protein interactomes due to infection progression, virus diversity, and host range and plasticity, two different but complementary experimental approaches would be required. One approach consists of protein complementation assays, e.g., yeast two-hybrid ( $\mathrm{Y} 2 \mathrm{H})$ [26], the most commonly used technique. In these assays, the potential interaction is decontextualized, which can present some limitations, but offers a sensitive and cost-effective mean to screen a library of proteins fragments prepared from the de-sired cell types, tissues or entire organisms against a viral protein of interest. Y2H has led to the distillation of multiple protein interactomes, including the construction of a comprehensive virus-host interactome for two members of the family Herpesviridae [3]. The cellular decontextualization of interactions associated to the $\mathrm{Y} 2 \mathrm{H}$ technique can in part be overcome by the use of bimolecular fluorescence complementation (BiFC) [27], which allows identifying protein-protein inter-actions in the real host cell, and also provides valuable information about subcellular localization of the complexes and its potential change during the course of infection. Unfortunately, this technique is hard to scale up to allow for highthroughput analyses of hundreds (or even thousands) of potential interactions. The other approach consists of protein purification assays, either by co-immunoprecipitation (co-IP) [28], provided the availability of a suitable antibody, by affinity purification (AP) of properly tagged proteins [29], or by intact cell labeling with chemical cross-linkers, the protein interaction reporters (PIRs) [30], followed by mass spectrometry (MS). These techniques offer a high-throughput way of capturing, from cell extracts, all proteins that inter-act in form 
of complexes with the viral protein thanks to mass spectroscopy. In particular, AP-MS has allowed the construction of the global interactome of HIV [31], and also the identification of the host targets of some plant virus proteins during real infection [32]. The PIR technol-ogy has also allowed the identification of host targets for a plant virus, but has additionally provided valuable information on the structural do-mains involved in the interactions [33]. The future use of recently developed protein microarrays also constitutes a plausible strategy for the discovery of virus-host interactions at a large scale [34]. Protein purification followed by MS appears hence suitable to analyze in a high-throughput way the interactome along the three dimensions, although at the cost of having false positives. However, protein complementation seems constrained to only explore in a high-throughput way the dimension of virus genotype. In this regard, gene expression profiling at different times of infection could help to interpret in a dynamic way protein-protein interaction data [35]. Certainly, an integrative analysis of multi-omic data would be required to accurately assess the plastodynamic nature of virus-host interactions, and ultimately compose a detailed model of viral infection [7].

In sum, the information resulting from this type of studies would allow explaining different stages of infection, and also different susceptibilities between individuals, with applications from personalized medicine to epidemiology [36]. This would also be a valuable framework to shed light on the intricate mechanisms that operate during the virus-host coevolution [20], as it would help to identify commonalities and specificities of interactions that arise after genetic diversification (of vi-ruses and hosts) in scenarios of colonization and of adaptation to new hosts $[37,38]$. In this sense, it might serve to study emerging infectious diseases. In addition, it would ground the foundations to develop in the mid-term future new effective antiviral therapies that either block the interaction of a viral protein with an essential cellular target at a precise time (according to the infection progression state), or, alternatively, interfere at a given point of the cellular network (perhaps tightly host specific) to counteract the effect of the viral quasispecies [39,40]. The potential development of such therapies, which would limit side effects on cell physiology and viral escapes, is especially relevant in the current global scenario where multiple viruses are threatening our health and behavior.

\section{Acknowledgement}

This work is supported by the grants BFU2015-66894-P (to G.R.), BIO2014-54269-R (to JA.D.) and BFU2015-65037-P (to S.F.E.) from the Ministerio de Economía, Industria y Competitividad, and by the grant PROMETEOII/2014/021 from the Generalitat Valenciana (to S.F.E. and J-A.D.).

\section{References}

[1] B. de Chassey, V. Navratil, L. Tafforeau, M.S. Hiet, A. Aublin-Gex, S. Agaugué, G. Meiffren, F. Pradezynski, B.F. Faria, T. Chantier, M. Le Breton, J. Pellet, N. Davoust, P.E. Mangeot, A. Chaboud, F. Penin, Y. Jacob, P.O. Vidalain, M. Vidal, P. André, C. RabourdinCombe, V. Lotteau, Hepatitis C virus infection protein network, Mol. Syst. Biol. 4 (2008) 230.

[2] A. Pichlmair, K. Kandasamy, G. Alvisi, O. Mulhern, R. Sacco, M. Habjan, M. Binder, A. Stefanovic, C.A. Eberle, A. Goncalves, T. Bürckstümmer, A.C. Müller, A. Fauster, C. Holze, K. Lindsten, S. Goodbourn, G. Kochs, F. Weber, R. Bartenschlager, A.G. Bowie, K.L. Bennett, J. Colinge, G. Superti-Furga, Viral immune modulators perturb the human molecular network by common and unique strategies, Nature 487 (2012) 486-490. 
[3] P. Uetz, Y.A. Dong, C. Zeretzke, C. Atzler, A. Baiker, B. Berger, S.V. Rajagopala, M. Roupelieva, D. Rose, E. Fossum, J. Haas, Herpesviral protein networks and their in-teraction with the human proteome, Science 311 (2006) 239-242.

[4] S.L. Tan, G. Ganji, B. Paeper, S. Proll, M.G. Katze, Systems biology and the host response to viral infection, Nat. Biotechnol. 25 (2007) 1383-1389.

[5] C.C. Friedel, J. Haas, Virus-host interactomes and global models of virus-infected cells, Trends Microbiol. 19 (2011) 501-508.

[6] S.F. Elena, G. Rodrigo, Towards an integrated molecular model of plant-virus interactions, Curr. Opin. Virol. 2 (2012) 719-724.

[7] G.L. Law, M.J. Korth, A.G. Benecke, M.G. Katze, Systems virology: host-directed approaches to viral pathogenesis and drug targeting, Nat. Rev. Microbiol. 11 (2013) 455-466.

[8] A.L. Barabási, N. Gulbahce, J. Loscalzo, Network medicine: a network-based approach to human disease, Nat. Rev. Genet. 12 (2011) 56-68.

[9] J.N. Culver, M.S. Padmanabhan, Virus-induced disease: altering host physiology one interaction at a time, Annu. Rev. Phytopathol. 45 (2007) 221-243.

[10] F. Kirchhoff, Immune evasion and counteraction of restriction factors by HIV-1 and other primate lentiviruses, Cell Host Microbe 8 (2010) 55-67.

[11] D. Yoon, H. Kim, H. Suh-Kim, R.W. Park, K. Lee, Differentially co-expressed interacting protein pairs discriminate samples under distinct stages of HIV type 1 infection, BMC Syst. Biol. 5 (2011) S1.

[12] E.J.D. Greenwood, N.J. Matheson, K. Wals, D.J.H. van den Boomen, R. Antrobus, J.C. Williamson, P.J. Lehner, Temporal proteomic analysis of HIV infection reveals remodeling of the phosphoproteome by lentiviral Vif variants, eLife 5 (2016), e18296. .

[13] I.W. Taylor, R. Linding, D. Warde-Farley, Y. Liu, C. Pesquita, D. Faria, S. Bull, T. Pawson, Q. Morris, J.L. Wrana, Dynamic modularity in protein interaction networks predicts breast cancer outcome, Nat. Biotechnol. 27 (2009) 199-204.

[14] S.Y. Kim, R. Byrn, J. Groopman, D. Baltimore, Temporal aspects of DNA and RNA syn-thesis during human immunodeficiency virus infection: evidence for differential gene expression, J. Virol. 63 (1989) 3708-3713.

[15] R. Sanjuán, A. Moya, S.F. Elena, The distribution of fitness effects caused by singlenucleotide substitutions in an RNA virus, Proc. Natl. Acad. Sci. U. S. A. 101 (2004) 83968401.

[16] P. Carrasco, F. de la Iglesia, S.F. Elena, Distribution of fitness and virulence effects caused by single-nucleotide substitutions in Tobacco etch virus, J. Virol. 81 (2007) 1297912984.

[17] A. Acevedo, L. Brosdsky, R. Andino, Mutational and fitness landscapes of an RNA virus revealed through population sequencing, Nature 505 (2014) 686-690.

[18] R. Andino, E. Domingo, Viral quasispecies, Virology 479 (2015) 46-51.

[19] T. Okamoto, H. Omori, Y. Kaname, T. Abe, Y. Nishimura, T. Suzuki, T. Miyamura, T. Yoshimori, K. Moriishi, Y. Matsuura, A single-amino-acid mutation in hepatitis C virus NS5A disrupting FKBP8 interaction impairs viral replication, J. Virol. 82 (2008) 3480-3489.

[20] M.D. Daugherty, H.S. Malik, Rules of engagement: molecular insights from host-virus arms races, Annu. Rev. Genet. 46 (2012) 677-700.

[21] S.S. Gervasi, D.J. Civitello, H.J. Kilvitis, L.B. Martin, The context of host competence: a role for plasticity in host-parasite dynamics, Trends Parasitol. 31 (2015) 419425.

[22] J. Hillung, F. García-García, J. Dopazo, J.M. Cuevas, S.F. Elena, The transcriptomics of an experimentally evolved plant-virus interaction, Sci. Rep. 6 (2016) 24901. 
[23] A. Sen, A.J. Pruijssers, T.S. Dermody, A. García-Sastre, H.B. Greenberg, The early inter-feron response to rotavirus is regulated by PKR and depends on MAVS/IPS-1, RIG-I, MDA5, and IRF3, J. Virol. 85 (2011) 3717-3732.

[24] P. Domingues, F. Golebiowski, M.H. Tatham, A.M. Lopes, A. Taggart, R.T. Hay, B.G. Hale, Global reprogramming of host SUMOylation during influenza virus infection, Cell Rep. 13 (2015) 1467-1480.

[25] A. Greco, L. Arata, E. Soler, X. Gaume, Y. Couté, S. Hacot, A. Callé, K. Monier, A.L. Epstein, J.-C. Sanchez, P. Bouvet, J.-J. Diaz, Nucleolin interacts with US11 protein of herpes simplex virus 1 and is involved in its trafficking, J. Virol. 86 (2012) 1449-1457.

[26] A.J. Walhout, M. Vidal, High-throughput yeast two-hybrid assays for large-scale protein interaction mapping, Methods 24 (2001) 297-306.

[27] E.P. Pratt, J.L. Owens, G.H. Hockerman, C.D. Hu, Bimolecular fluorescence complementation (BiFC) analysis of protein-protein interactions and assessment of subcel-lular localization in live cells, Methods Mol. Biol. 1474 (2016) 153-170.

[28] J.J. Moresco, P.C. Carvalho, J.R. Yates, Identifying components of protein complexes in C. elegans using co-immunoprecipitation and mass spectrometry, J. Proteomics 73 (2010) 2198-2204.

[29] W.H. Dunham, M. Mullin, A.C. Gingras, Affinity-purification coupled to mass spectrometry: basic principles and strategies, Proteomics 12 (2012) 1576-1590.

[30] H. Zhang, X. Tang, G.R. Munske, N. Tolic, G.A. Anderson, J.E. Bruce, Identification of protein-protein interactions and topologies in living cells with chemical cross-linking and mass spectrometry, Mol. Cell. Proteomics 8 (2009) 409-420.

[31] S. Jäger, P. Cimermancic, N. Gulbahce, J.R. Johnson, K.E. McGovern, S.C. Clarke, M. Shales, G. Mercenne, L. Pache, K. Li, H. Hernandez, G.M. Jang, S.L. Roth, E. Akiva, J. Marlett, M. Stephens, I. D'Orso, J. Fernandes, M. Fahey, C. Mahon, A.J. O' Donoghue, A. Todorovic, J.H. Morris, D.A. Maltby, T. Alber, G. Cagney, F.D. Bushman, J.A. Young, S.K. Chanda, W.I. Sundquist, T. Kortemme, R.D. Hernandez, C.S. Craik, A. Burlingame, A. Sali, A.D. Frankel, N.J. Krogan, Global landscape of HIV-human protein complexes, Nature 481 (2012) 365-370.

[32] F. Martínez, G. Rodrigo, V. Aragonés, M. Ruiz, I. Lodewijck, U. Fernández, S.F. Elena, J.A. Daròs, Interaction network of tobacco etch potyvirus NIa protein with the host proteome during infection, BMC Genomics 17 (2016) 87.

[33] S.L. DeBlasio, J.D. Chavez, M.M. Alexander, J. Ramsey, J.K. Eng, J. Mahoney, S.M. Gray, J.E. Bruce, M. Cilia, Visualization of host-polerovirus interaction topologies using protein interaction reporter technology, J. Virol. 90 (2016) 1973-1987.

[34] J. Yazaki, M. Galli, A.Y. Kim, K. Nito, F. Aleman, K.N. Chang, A.R. Carvunis, R. Quan, H. Nguyen, L. Song, J.M. Alvarez, S.S. Huang, H. Chen, N. Ramachandran, S. Altmann, R.A. Gutiérrez, D.E. Hill, J.I. Schroeder, J. Chory, J. LaBaer, M. Vidal, P. Braun, J.R. Ecker, Mapping transcription factor interactome networks using HaloTag protein ar-rays, Proc. Natl. Acad. Sci. U. S. A 113 (2016) E4238-E4247.

[35] S.D. Shapira, I. Gat-Viks, B.O.V. Shum, A. Dricot, M.M. de Grace, L. Wu, P.B. Gupta, T. Hao, S.J. Silver, D.E. Root, D.E. Hill, A. Regev, N. Hacohen, A physical and regulatory

map of host-influenza interactions reveals pathways in H1N1 infection, Cell 139 (2009) 1255-1267.

[36] A.D. Weston, L. Hood, Systems biology, proteomics, and the future of health care: toward predictive, preventative, and personalized medicine, J. Proteome Res. 3 (2004) 179-196. [37] P.N. Dodds, G.J. Lawrence, A.M. Catanzariti, T. Teh, C.I. Wang, M.A. Ayliffe, B. Kobe, J.G. Ellis, Direct protein interaction underlies gene-for-gene specificity and coevolu- 
tion of the flax resistance genes and flax rust avirulence genes, Proc. Natl. Acad. Sci. U. S. A. 103 (2006) 8888-8893.

[38] G. Rodrigo, J. Carrera, V. Ruiz-Ferrer, F.J. del Toro, C. Llave, O. Voinnet, S.F. Elena, A meta-analysis reveals the commonalities and differences in Arabidopsis thaliana re-sponse to different viral pathogens, PLoS One 7 (2012), e40526. .

[39] P. Farci, A. Shimoda, A. Coiana, G. Diaz, G. Peddis, J.C. Melpolder, A. Strazzera, D.Y. Chien, S.J. Munoz, A. Balestrieri, R.H. Purcell, H.J. Alter, The outcome of acute hepatitis C predicted by the evolution of the viral quasispecies, Science 288 (2000) 339-344.

[40] T.K. Scheel, C.M. Rice, Understanding the hepatitis C virus life cycle paves the way for highly effective therapies, Nat. Med. 19 (2013) 837-849. 


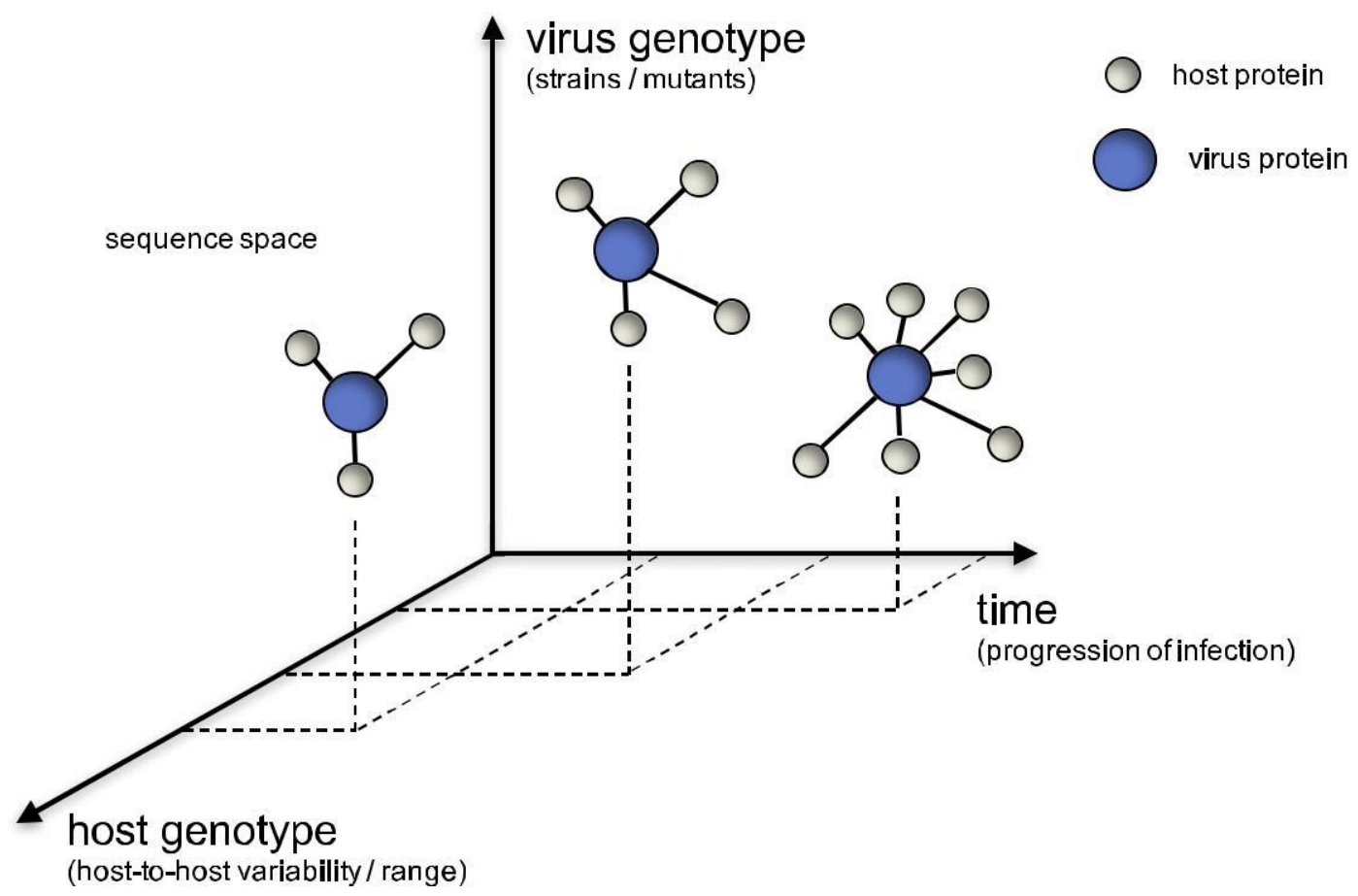

Fig. 1. Scheme of the plastodynamic virus-host interactome. The protein interactome can vary with time (i.e., as long as the virus infects the organism), with the sequence of the virus that codes for interacting proteins (this has a short-term effect as viruses are replicated by errorprone polymerases, and a long-term effect for the emergence of new strains), and finally with the host (as different genotypes will deploy different proteomes with particular interaction abilities). 